\title{
The Decay Data Evaluation Project (DDEP) and the JEFF-3.3 radioactive decay data library: Combining international collaborative efforts on evaluated decay data
}

\author{
Mark A. Kellett ${ }^{1, \mathrm{a}}$ and Olivier Bersillon ${ }^{2, \mathrm{~b}}$ \\ ${ }^{1}$ CEA, LIST, Laboratoire National Henri Becquerel (LNE-LNHB), Bât. 602 PC 111, CEA-Saclay, 91191 Gif-sur-Yvette \\ Cedex, France \\ 2 Retired from CEA, DAM Île-de-France, Bruyères-le-Châtel, 91297 Arpajon Cedex, France
}

\begin{abstract}
The Decay Data Evaluation Project (DDEP), is an international collaboration of decay data evaluators formed with groups from France, Germany, USA, China, Romania, Russia, Spain and the UK, mainly from the metrology community. DDEP members have evaluated over 220 radionuclides, following an agreed upon methodology, including a peer review. Evaluations include all relevant parameters relating to the nuclear decay and the associated atomic processes. An important output of these evaluations are recommendations for new measurements, which can serve as a basis for future measurement programmes. Recently evaluated radionuclides include: ${ }^{18} \mathrm{~F},{ }^{59} \mathrm{Fe},{ }^{82} \mathrm{Rb},{ }^{82} \mathrm{Sr},{ }^{88} \mathrm{Y},{ }^{90} \mathrm{Y},{ }^{89} \mathrm{Zr},{ }^{94 m} \mathrm{Tc},{ }^{109} \mathrm{Cd},{ }^{133} \mathrm{Ba},{ }^{140} \mathrm{Ba}$, ${ }^{140} \mathrm{La},{ }^{151} \mathrm{Sm}$ and ${ }^{169} \mathrm{Er}$.

The DDEP recommended data have recently been incorporated into the JEFF-3.3 Radioactive Decay Data Library. Other sources of nuclear data include 900 or so radionuclides converted from the Evaluated Nuclear Structure Data File (ENSDF), 500 from two UK libraries (UKPADD6.12 and UKHEDD2.6), the IAEA Actinide Decay Data Library, with the remainder converted from the NUBASE evaluation of nuclear properties. Mean decay energies for a number of radionuclides determined from total absorption gammaray spectroscopy (TAGS) have also been included, as well as more recent European results from TAGS measurements performed at the University of Jyväskylä by groups from the University of Valencia, Spain and SUBATECH, the University of Nantes, France.

The current status of the DDEP collaboration and the JEFF Radioactive Decay Data Library will be presented.
\end{abstract}

\section{Introduction}

The Decay Data Evaluation Project (DDEP) [1], is an international collaboration founded in 1995, following an initial agreement in 1994 between the Laboratoire National Henri Becquerel (LNE-LNHB), France, and the Physikalisch-Technische Bundesanstalt (PTB), Germany, both of which are the recognised National Metrology Institutes (NMIs) for ionising radiation in their respective countries. The collaboration was formally established with groups from the US, and subsequently joined by groups from China, Romania, Russia, Spain and the UK.

At present the collaboration undertakes the evaluation of decay scheme data for well-chosen radionuclides in the area of radionuclide metrology, detector calibration, nuclear medicine, both diagnostic and pharmaceutical, as well as in response to user requests from the wider community, and in cooperation with activities of the International Atomic Energy Agency (IAEA). To date the DDEP members have evaluated over 220 radionuclides, following an agreed upon methodology, including a peer review. Evaluations include all parameters relating to the nuclear decay; radiation energies and

\footnotetext{
a e-mail: mark.kellett@cea.fr

${ }^{b}$ Deceased, 29 November 2016
}

intensities, half-life, branching fractions, etc., as well as the associated atomic data; X-rays, Auger electrons, conversion electrons, etc. An important output of these evaluations are recommendations for new measurements, which can serve as a basis for future measurement programmes. Recently evaluated radionuclides include: ${ }^{18} \mathrm{~F},{ }^{52} \mathrm{Mn},{ }^{52 m} \mathrm{Mn},{ }^{59} \mathrm{Fe},{ }^{76} \mathrm{Br},{ }^{82} \mathrm{Rb},{ }^{82} \mathrm{Sr},{ }^{88} \mathrm{Y},{ }^{90} \mathrm{Y},{ }^{89} \mathrm{Zr}$, ${ }^{94 m} \mathrm{Tc},{ }^{109} \mathrm{Cd},{ }^{133} \mathrm{Ba},{ }^{140} \mathrm{Ba},{ }^{140} \mathrm{La},{ }^{151} \mathrm{Sm}$ and ${ }^{169} \mathrm{Er}$.

The DDEP recommended data have recently been incorporated into the JEFF Radioactive Decay Data Library, which forms part of the suite of nuclear data libraries produced under the auspices of the Joint Evaluated Fission and Fusion (JEFF) file project of the OECD Nuclear Energy Agency [2]. Following on from previous versions of the radioactive decay data library, a recent update, JEFF-3.3, is now available. Other sources of nuclear data, which contribute to this library, have been re-visited and updated. Specifically the 900 or so radionuclides converted from the Evaluated Nuclear Structure Data File (ENSDF) [3] have been updated, where necessary, along with new evaluations from the two UK libraries (UKPADD-6.12 and UKHEDD-2.6 [4]), the IAEA Actinide Decay Data Library [5] with the remainder converted from the NUBASE evaluation of nuclear properties [6]. In accordance with the assessment of fission product decay data for decay heat calculations 
undertaken by sub-group 25 of the OECD/NEA Working Party on International Nuclear Data Evaluation Cooperation (WPEC) [7], mean decay energies for a number of radionuclides determined from total absorption gammaray spectroscopy (TAGS) have also been included [8], as well as more recent European results from TAGS measurements performed at the University of Jyväskylä, Finland, by groups from the University of Valencia, Spain $[9,10]$ and SUBATECH, the University of Nantes, France [11].

\section{The decay data evaluation project}

\subsection{Context}

Within the metrology community, in the early 1990s, there was a specific need identified for evaluated decay scheme data for use in absolute activity measurements, including important atomic data, e.g., conversion electrons, X-rays, Auger-electrons. During international inter-comparison exercises, differences in recommended activity values were seen to be dependent upon the decay scheme data used. Hence, the international DDEP group was established in 1995, in which a common evaluation methodology was defined and adopted, including a comprehensive review process. The Bureau International des Poids et Mesures (BIPM), which, through the Consultative Committee on Ionising Radiation, oversees the international metrology community inter-comparison exercises, recommended that the DDEP evaluated decay scheme data be used by all metrology institutes. As such these evaluations are compiled and edited by the LNELNHB and published as volumes of BIPM Monographie5 [12-19].

\subsection{Mission}

The DDEP aims to provide recommended decay data to non-specialists. Typical users are not nuclear structure experts, they are generally metrologists working in the field of alpha- and gamma-spectrometry, or with liquid scintillation techniques. Thus the goal of the DDEP is to provide all of the relevant data, i.e., half-life, energy and emission intensities for the main decay radiations (alpha, beta and gamma) and the associated atomic data due to the internal conversion process, i.e., energy and emission intensities for X-rays, conversion electrons, and Auger electrons, and to facilitate the access to these data in as convenient a way as possible. In this respect, the DDEP does not aim to compete with the more nuclear structure oriented evaluations from the ENSDF project, but sees itself as a complementary effort focussed on producing recommended decay data relevant to the wider user community. For example, DDEP members often use, as a starting point in building the decay scheme, the adopted nuclear levels provided in the ENSDF database, as information from reaction data is also used in their determination. A close working relationship is maintained between the two projects, with members regularly attending the meetings of the other.

\subsection{Members}

Since the inception of the project, the LNE-LNHB has produced the tables of recommended data and edited the
Table 1. Current members of the Decay Data Evaluation Project.

\begin{tabular}{|c|c|}
\hline Member & Affiliation \\
\hline $\begin{array}{l}\text { Mark A. Kellett Coordinator } \\
\text { Xavier Mougeot } \\
\text { Christophe Dulieu IT support }\end{array}$ & LNE-LNHB, France \\
\hline $\begin{array}{l}\text { Arzu Arinc } \\
\text { Andy Pearce }\end{array}$ & NPL, UK \\
\hline $\begin{array}{l}\text { Valery P. Chechev } \\
\text { Nikolai Kuzmenko }\end{array}$ & KRI, Russia \\
\hline Mónica Galán & СТВТО \\
\hline Xiaolong Huang & CIAE, China \\
\hline Aurelian Luca & IFIN-HH, Romania \\
\hline Alan L. Nichols & Surrey Uni., UK \\
\hline Members joining in 2016 : & \\
\hline Herbert Janssen & PTB, Germany \\
\hline Haoran Liu & NIM, China \\
\hline Brian Zimmerman & NIST, USA \\
\hline
\end{tabular}

Monographie BIPM 5 publications, and since mid-2012 the LNE-LNHB are coordinating the project, including the review process. The currently active members are given in Table 1. Following a "call to arms" at the International Radionuclide Metrology Conference (ICRM) in 2015, the training of two new members from NIST (USA) and the NIM (China) is in progress, with a new member from PTB (Germany) also joining.

\subsection{Evaluation procedure}

All published values are examined and analysed (assuming there are some), during which certain values may be rejected or have their uncertainties adjusted. Once this is done, a statistical analysis can be undertaken in order to determine the recommended value, hence the final decay scheme will be based on available measured values - as far as possible.

In order to build a complete decay scheme, basic physical constraints are applied:

1. The sum of the branching fractions for all decay modes from the parent nuclide should equal $100 \%$.

2. The sum of the transition probabilities of all radiation types arriving at the ground state of the daughter(s) should equal $100 \%$.

3. The transition probability to each level should be equal to the transition probability from that level, i.e. population balance of each level.

4. Total decay energy should be conserved, such that the sum of all of the decay radiations is equal to the $Q$-value.

The transition intensities for all $\gamma$-rays are calculated from the recommended emission intensities and the internal conversion coefficients, which are interpolated using the BrIcc code [20]. These also allow the associated atomic data to be calculated by using the relevant $\omega_{K}$ and $\omega_{L}$ values and other relevant parameters. A comparison of the calculated X-ray data with measured data (if they exist) is always made.

The recently developed BETASHAPE code [21] is now being used to calculate the $\log f t$ values and the mean beta energies included in the DDEP evaluations. 


\subsection{Publications}

Apart from publishing evaluations through the Bureau International des Poids et Mesures (BIPM) in the Monographie series BIPM-5, other derived products have also been created by the LNE-LNHB, including the Mini Table of Radionuclides 2015 [22]. This pocket guide includes all of the main decay radiations for 296 radionuclides, commonly found in research laboratories, industry and the medical profession. The guide is published through EDP Sciences and during the first ten months after publication, 630 copies were sold.

The two most recent volumes in the Monographie BIPM-5 series contain evaluations for the following radionuclides:

volume 7 [18], published in 2013, contains 29 radionuclides: ${ }^{14} \mathrm{C},{ }^{35} \mathrm{~S},{ }^{36} \mathrm{Cl},{ }^{37} \mathrm{Ar},{ }^{45} \mathrm{Ca},{ }^{67} \mathrm{Ga},{ }^{68} \mathrm{Ga}$, ${ }^{68} \mathrm{Ge},{ }^{127} \mathrm{Sb},{ }^{127} \mathrm{Te},{ }^{127 m} \mathrm{Te},{ }^{134} \mathrm{Cs},{ }^{141} \mathrm{Ce},{ }^{147} \mathrm{Nd}$, ${ }^{147} \mathrm{Pm},{ }^{195} \mathrm{Au},{ }^{206} \mathrm{Hg},{ }^{207} \mathrm{Tl},{ }^{208} \mathrm{Tl},{ }^{209} \mathrm{Tl},{ }^{211} \mathrm{~Pb},{ }^{211} \mathrm{At}$, ${ }^{213} \mathrm{Bi},{ }^{215} \mathrm{Bi},{ }^{228} \mathrm{Th},{ }^{242} \mathrm{Cm},{ }^{243} \mathrm{Cm},{ }^{244} \mathrm{Cm}$ and ${ }^{245} \mathrm{Cm}$,

volume 8 [19], published in 2016, contains 32 radionuclides: ${ }^{41} \mathrm{Ca},{ }^{47} \mathrm{Sc},{ }^{52} \mathrm{Fe},{ }^{58} \mathrm{Co},{ }^{61} \mathrm{Cu},{ }^{63} \mathrm{Zn}$, ${ }^{73} \mathrm{Se},{ }^{82} \mathrm{Rb},{ }^{82} \mathrm{Sr},{ }^{88} \mathrm{Y},{ }^{89} \mathrm{Zr},{ }^{93} \mathrm{Zr},{ }^{93 m} \mathrm{Nb},{ }^{94 m} \mathrm{Tc}$, ${ }^{106} \mathrm{Ru},{ }^{106} \mathrm{Rh},{ }^{109} \mathrm{Cd},{ }^{131} \mathrm{I},{ }^{127} \mathrm{Xe},{ }^{131 m} \mathrm{Xe},{ }^{133} \mathrm{Ba}$, ${ }^{140} \mathrm{Ba},{ }^{138} \mathrm{La},{ }^{140} \mathrm{La},{ }^{144} \mathrm{Ce},{ }^{144} \mathrm{Pr},{ }^{144 m} \mathrm{Pr},{ }^{148} \mathrm{Pm}$, ${ }^{148 m} \mathrm{Pm},{ }^{151} \mathrm{Sm},{ }^{169} \mathrm{Er}$ and ${ }^{198} \mathrm{Au}$.

In connection with the decay data evaluation work, some members of the DDEP were asked to contribute an article to a special issue of Metrologia on "Uncertainties in Nuclear Decay Data Evaluations" [23].

\subsection{International contribution}

A number of international initiatives have involved the participation of DDEP members and have adopted the evaluation methodology developed with the DDEP. For example, members of the DDEP were active in numerous IAEA Coordinated Research Projects (CRPs), including the CRP on the "Update of X Ray and Gamma Ray Decay Data Standards for Detector Calibration and Other Applications", which published the final report of recommended data in 2007 [24] and the later project which produced the "Library of Recommended Actinide Decay Data, 2011" [5].

Two ongoing IAEA CRPs also involve a number of DDEP members producing evaluations of recommended decay data. For example, the CRP on "Nuclear Data for Charged-particle Monitor Reactions and Medical Isotope Production" [25], see for example [26], and the CRP on "Testing and Improving the International Reactor Dosimetry and Fusion File (IRDFF)", see for example [27].

\subsection{Medical domain}

In connection with the IAEA CRP on "Nuclear Data for Charged-particle Monitor Reactions and Medical Isotope Production" [25] the participating DDEP members have so far completed evaluations for:

Monitor reactions: ${ }^{61} \mathrm{Cu}$ and ${ }^{63} \mathrm{Zn}$.

Positron emitters: ${ }^{52} \mathrm{Fe},{ }^{52} \mathrm{Mn},{ }^{52 m} \mathrm{Mn},{ }^{64} \mathrm{Cu},{ }^{73} \mathrm{Se}$, ${ }^{76} \mathrm{Br},{ }^{89} \mathrm{Zr}$ and ${ }^{94 m} \mathrm{Tc}$.
Therapeutic alpha emitters: ${ }^{230} \mathrm{U}$ decay chain: ${ }^{218} \mathrm{Rn}$, ${ }^{214} \mathrm{Po},{ }^{210} \mathrm{~Pb},{ }^{210} \mathrm{Bi},{ }^{210} \mathrm{Po}$ with ${ }^{230} \mathrm{U},{ }^{226} \mathrm{Th},{ }^{222} \mathrm{Ra}$ in progress.

Other evaluations have been completed, but are awaiting new calculations of the X-ray and Auger electron data:

Diagnostic gamma emitters: ${ }^{99} \mathrm{Mo} /{ }^{99 m} \mathrm{Tc}$ and ${ }^{111} \mathrm{In}$. Auger-electron and X-ray emitters: ${ }^{103} \mathrm{Pd},{ }^{131} \mathrm{Cs}$.

\subsection{Access to decay scheme data}

The DDEP evaluations are placed on the website developed and hosted by the LNE-LNHB and available at http://www.nucleide.org. Included on this website are two PDF files which contain the data "Tables" showing the recommended values for the decay scheme parameters and a "Comments" file from the evaluator. This latter file includes complete details provided by the evaluator on the procedure undertaken to arrive at the recommended values, including data selection and/or rejection, adjustment of uncertainties, etc. and may also include recommendations for future measurements when the available experimental data are not deemed appropriate to enable a balanced decay scheme to be constructed. Three additional computer readable ASCII files are also made available in different formats: an ENSDF file, a PENNUC file and a simple comma separated values (CSV) file. Further details on the availability of the data and the online access tool LARAWEB can be found in the recent publication of Dulieu et al. [28].

\section{The JEFF radioactive decay data library}

\subsection{Background}

The JEF-2.2 library, released in the early 1990s, was, for many years, the reference decay data library within Europe. However, identified shortcomings led to a change in strategy for the next generation of decay data libraries within the JEFF project. Hence, JEFF-3.0 was constructed using a completely new approach compared to JEF2.2. A skeleton based on NUBASE was agreed upon, whereby all relevant radionuclides were identified. More detailed evaluations from specialised evaluated decay data libraries (UKHEDD, UKPADD, DDEP, IAEA, etc.), with also a contribution from ENSDF, were then used to replace individual radionuclides. Only evaluations having an energy balance better than $2 \%$ were taken from ENSDF, thus differing from the philosophy adopted for ENDF/BVII, where almost all evaluations are taken from ENSDF without this quality specification.

The first major release, post JEF-2.2, was the JEFF3.1 library of May 2005, which included 3852 nuclei, of which 226 are stable. It was also essential to ensure that the decay daughter(s) from any given nuclei would also be present, ensuring that complete decay chains could be formed throughout. Limited testing showed problems with decay heat prediction, mainly due to the pandemonium effect [29], i.e., missing beta feeding to the higher energy levels, hence missing gamma energy, and increased mean beta energy. 
Table 2. Nuclei updated in JEFF-3.1.1 using Greenwood et al.'s [8] TAGS mean energy values (in MeV). (An uncertainty of $10 \%$ was applied to the adopted mean energies.)

\begin{tabular}{rcccc}
\hline & JEFF-3.1 & TAGS & JEFF-3.1 & TAGS \\
\hline Nuclide & \multicolumn{2}{c}{$\langle\beta\rangle$} & \multicolumn{2}{c}{$\langle\gamma\rangle$} \\
\hline${ }^{9} \mathrm{Rb}$ & 2.049 & 1.916 & 1.982 & 2.270 \\
${ }^{90 m} \mathrm{Rb}$ & 1.403 & 1.118 & 3.240 & 3.869 \\
${ }^{91} \mathrm{Rb}$ & 1.612 & 1.368 & 2.269 & 2.706 \\
${ }^{95} \mathrm{Sr}$ & 2.208 & 1.901 & 1.118 & 1.790 \\
${ }^{139} \mathrm{Cs}$ & 1.640 & 1.671 & 0.345 & 0.305 \\
${ }^{141} \mathrm{Cs}$ & 1.935 & 1.506 & 0.770 & 1.701 \\
${ }^{143} \mathrm{Ba}$ & 1.417 & 1.195 & 1.417 & 1.341 \\
${ }^{144} \mathrm{Ba}$ & 1.040 & 0.930 & 1.040 & 0.785 \\
${ }^{145} \mathrm{Ba}$ & 1.860 & 1.285 & 1.860 & 1.833 \\
${ }^{142} \mathrm{La}$ & 0.868 & 0.962 & 2.325 & 2.121 \\
${ }^{143} \mathrm{La}$ & 1.237 & 1.235 & 0.252 & 0.439 \\
${ }^{144} \mathrm{La}$ & 1.382 & 0.986 & 2.330 & 3.085 \\
${ }^{145} \mathrm{La}$ & 1.499 & 0.762 & 0.624 & 2.144 \\
${ }^{148} \mathrm{Ce}$ & 0.713 & 0.586 & 0.713 & 0.486 \\
${ }^{147} \mathrm{Pr}$ & 0.899 & 0.669 & 0.899 & 0.929 \\
${ }^{148} \mathrm{Pr}$ & 1.679 & 1.348 & 0.938 & 1.776 \\
${ }^{148 m} \mathrm{Pr}$ & 1.701 & 1.239 & 0.937 & 2.050 \\
${ }^{149} \mathrm{Pr}$ & 1.286 & 0.811 & 0.305 & 1.332 \\
${ }^{151} \mathrm{Pr}$ & 1.394 & 1.114 & 1.394 & 1.363 \\
${ }^{153} \mathrm{Nd}$ & 1.112 & 1.153 & 1.112 & 0.509 \\
${ }^{154} \mathrm{Nd}$ & 0.937 & 0.856 & 0.937 & 0.551 \\
${ }^{155} \mathrm{Nd}$ & 1.500 & 1.085 & 1.500 & 1.543 \\
${ }^{152} \mathrm{Pm}$ & 1.326 & 1.304 & 0.285 & 0.345 \\
${ }^{153} \mathrm{Pm}$ & 0.686 & 0.663 & 0.073 & 0.123 \\
${ }^{154} \mathrm{Pm}$ & 1.321 & 0.849 & 1.321 & 1.876 \\
${ }^{156} \mathrm{Pm}$ & 1.717 & 1.206 & 1.717 & 2.206 \\
${ }^{157} \mathrm{Pm}$ & 1.453 & 1.540 & 1.453 & 0.849 \\
${ }^{157} \mathrm{Sm}$ & 0.912 & 0.839 & 0.912 & 0.585 \\
\hline${ }^{158} \mathrm{Sm}$ & 0.667 & 0.512 & 0.667 & 0.590 \\
\hline & & & \multicolumn{2}{c}{}
\end{tabular}

\subsection{Inclusion of TAGS data and the JEFF-3.1.1 release}

The initial decay heat benchmarking of JEFF-3.1, along with the work of the OECD/NEA Working Party on International Evaluation Co-operation subgroup 25 (WPEC-25) [7] highlighted some problems in the individual prediction of the gamma and beta decay heat components, the total decay heat prediction being satisfactory. It was believed that mean energy values determined through Total Absorption Gamma-ray Spectroscopy (TAGS) experiments could help correct this problem. Hence a limited number of twenty-nine nuclei (see Table 2) had their mean energies updated from the high quality work of Greenwood et al. [8]. The nuclei updated were only those where a detailed evaluation was not available, and hence the nuclei measured by this group were considered. No detailed uncertainty information was available in this work, thus a rather conservative 10\% uncertainty was applied to all the adopted mean energies.

In addition to the insertion of the TAGS data, JEFF3.1.1 also benefited from the availability of a further fifty high quality detailed evaluations through the updating that had been made of the two libraries from the UK (UKPADD-6.7 and UKHEDD-2.5). Hence, mainly as a consequence of the initial decay heat benchmarking, a moderately revised version, JEFF-3.1.1, was released in November 2007.
Table 3. Nuclei updated in JEFF-3.3 using recently measured TAGS mean energy values (in $\mathrm{MeV}$ ) at the University of Jyväskylä by the Valencia $[9,10]$ and Nantes [11] groups. (Uncertainties not shown.)

\begin{tabular}{ccccc}
\hline & JEFF-3.1 & TAGS & JEFF-3.1 & TAGS \\
\hline Nuclide & \multicolumn{4}{c}{$\langle\beta\rangle$} \\
\hline \multicolumn{5}{c}{ Valencia } \\
\hline${ }^{105} \mathrm{Mo}$ & 1.922 & 1.049 & 0.552 & 2.407 \\
${ }^{104} \mathrm{Tc}$ & 1.595 & 0.931 & 1.890 & 3.229 \\
${ }^{105} \mathrm{Tc}$ & 1.310 & 0.764 & 0.671 & 1.825 \\
${ }^{106} \mathrm{Tc}$ & 1.906 & 1.457 & 2.191 & 3.132 \\
${ }^{107} \mathrm{Tc}$ & 1.920 & 1.263 & 0.515 & 1.822 \\
\hline \multicolumn{5}{c}{ Nantes } \\
\hline${ }^{92} \mathrm{Rb}$ & 3.639 & 3.496 & 0.170 & 0.464 \\
\hline \multicolumn{5}{c}{}
\end{tabular}

Table 4. Source libraries and the number of evaluations adopted in the JEFF-3.3 library.

\begin{tabular}{lr}
\hline Source library & Number \\
\hline NUBASE & 2295 \\
ENSDF & 849 \\
DDEP & 140 \\
UKPADD-6.12 & 441 \\
UKHEDD-2.6 & 59 \\
IAEA & 66 \\
IRDFF & 2 \\
\hline Total & 3852 \\
\hline
\end{tabular}

\subsection{New European TAGS data and the JEFF-3.3 release}

The WPEC-25 sub-group had concluded that further TAGS experiments were merited and a list of appropriate nuclei was formulated. As a consequence of this, TAGS measurements began to be performed at the University of Jyväskylä, Finland, initially by a group from the University of Valencia, Spain, who were later joined by a second group from SUBATECH, the University of Nantes, France. To date only a limited number of nuclei have been published [9-11], but other nuclei are under analysis and further measurements are in progress or planned. Table 3 lists the nuclei already published which have been included into the JEFF-3.3 library.

It should be noted that two additional nuclei $\left({ }^{101} \mathrm{Nb}\right.$ and ${ }^{102} \mathrm{Tc}$ ) were not updated in JEFF-3.3 from the measurements made by the Valencia group, due to the very large $(\sim 50 \%)$ uncertainty on the mean $\gamma$ energy for the first nuclide and the almost identical values compared to the JEFF-3.1.1 library measured for the second. Indeed, ${ }^{102} \mathrm{Tc}$ was specifically measured as a means of validating the TAGS methodology.

Following on from the established production methodology for the JEFF library, updated or newly available source libraries have been included. The number of evaluations taken from each of these available libraries is given in Table 4.

\subsection{Conclusions}

The Decay Data Evaluation Project (DDEP), originally established within the metrology community to evaluate 
decay scheme data for use in detector calibration and international activity comparison exercises, has to date produced over 220 complete decay scheme evaluations. A methodology ensures that data are reviewed and detailed comments are provided on the evaluation process, to ensure the traceability of the recommended data. Shortcomings in the available data are documented and often lead to new measurement recommendations. The associated atomic data are calculated using the best available fundamental parameters and are included in the final evaluation tables. The evaluations are available from the website of the LNE-LNHB, in various tabulated formats, and through the LARAWEB online database. They are also published through the BIPM and as the Mini Table of Radionuclides.

The Radioactive Decay Data library of the Joint Evaluated Fission and Fusion (JEFF) project has been updated, with the new release of JEFF-3.3. Evaluations have been taken from various sources, mainly European, with a number from the ENSDF database. Data for the mean $\beta$ and $\gamma$ energies have been included from total absorption gamma-ray spectroscopy (TAGS) measurements, some of which have been produced recently by groups from the University of Valencia, Spain and SUBATECH, the University of Nantes, France, working at the University of Jyväskylä, Finland. Further TAGS data will be included, where appropriate, into future releases. Testing of this new release will be undertaken and published in due course.

The dedication of the DDEP evaluators, past and present, is gratefully acknowledged. Their efforts in providing high-quality recommended values is of paramount importance to the user community. The recent experimental work using the TAGS technique by the Valencia and Nantes groups has been shown to be invaluable in resolving the decay heat problem. The work of these two groups is highly appreciated.

The JEFF-3.3 Radioactive Decay Data Library is dedicated to the memory of Olivier Bersillon, whose untimely passing coincided with the release of this library, on which he worked so tirelessly over the past few years. His significant contribution is sincerely acknowledged.

\section{References}

[1] R.G. Helmer, E. Browne, M.-M. Bé, Journal of Nuclear Science and Technology 39, 455 (2002)

[2] A.J. Koning, E. Bauge, C.J. Dean, E. Dupont, U. Fischer, R.A. Forrest, R. Jacqmin, H. Leeb, M.A. Kellett, R.W. Mills et al., J. Korean Phys. Soc. 59, 1057s (2011)

[3] M.R. Bhat, Evaluated Nuclear Structure Data File (ENSDF), in Proc. Intern. Conf. on Nuclear Data for Science and Technology, edited by S.M. Qaim (Springer-Verlag, Berlin, Germany, 1992), p. 817

[4] R.J. Perry, C.J. Dean, A.L. Nichols, Nucl. Data Sheets 120, 261 (2014)

[5] M.A. Kellett, A.L. Nichols, Library of Recommended Actinide Decay Data, 2011, IAEA-STI/PUB/1618 (International Atomic Energy Agency, 2013)

[6] G. Audi, O. Bersillon, J. Blachot, A.H. Wapstra, Nucl. Phys. A729, 3 (2003)

[7] W.P. on International Evaluation Co-operation of the NEA Nuclear Science Committee, Assessment of
Fission Product Decay Data for Decay Heat Calculations, Vol. 25 (OECD Nuclear Energy Agency, 2007)

[8] R.C. Greenwood, R.G. Helmer, M.H. Putnam, K.D. Watts, Nucl. Instrum. Methods Phys. Res. A390, 95 (1997)

[9] A. Algora, D. Jordan, J.L. Tain, B. Rubio, J. Agramunt, A.B. Perez-Cerdan, F. Molina, L. Caballero, E. Nacher, A. Krasznahorkay et al., Phys. Rev. Lett. 105, 202501 (2010)

[10] E. Valencia, J.L. Tain, A. Algora, J. Agramunt, B. Rubio, S. Rice, W. Gelletly, P. Regan, A.-A. Zakari-Issoufou, M. Fallot et al., Submitted for publication, preprint available from arXiv 1609, 06128v1 (2016)

[11] A.-A. Zakari-Issoufou, M. Fallot, A. Porta, A. Algora, J.L. Tain, E. Valencia, S. Rice, V.M. Bui, S. Cormon, M. Estienne et al., Phys. Rev. Lett. 115, 102503 (2015)

[12] M.-M. Bé, V. Chisté, C. Dulieu, E. Browne, V. Chechev, N. Kuzmenko, R. Helmer, A. Nichols, E. Schönfeld, R. Dersch, Table of Radionuclides, Vol. 1 of Monographie BIPM-5 (Bureau International des Poids et Mesures, Pavillon de Breteuil, 92310 Sèvres, France, 2004)

[13] M.-M. Bé, V. Chisté, C. Dulieu, E. Browne, V. Chechev, N. Kuzmenko, R. Helmer, A. Nichols, E. Schönfeld, R. Dersch, Table of Radionuclides, Vol. 2 of Monographie BIPM-5 (Bureau International des Poids et Mesures, Pavillon de Breteuil, 92310 Sèvres, France, 2004)

[14] M.-M. Bé, V. Chisté, C. Dulieu, E. Browne, C. Baglin, V. Chechev, N. Kuzmenko, R. Helmer, F. Kondev, D. MacMahon et al., Table of Radionuclides, Vol. 3 of Monographie BIPM-5 (Bureau International des Poids et Mesures, Pavillon de Breteuil, 92310 Sèvres, France, 2006)

[15] M.-M. Bé, V. Chisté, C. Dulieu, E. Browne, V. Chechev, N. Kuzmenko, F. Kondev, A. Luca, M. Galán, A. Pearce et al., Table of Radionuclides, Vol. 4 of Monographie BIPM-5 (Bureau International des Poids et Mesures, Pavillon de Breteuil, 92310 Sèvres, France, 2008)

[16] M.-M. Bé, V. Chisté, C. Dulieu, X. Mougeot, E. Browne, V. Chechev, N. Kuzmenko, F. Kondev, A. Luca, M. Galán et al., Table of Radionuclides, Vol. 5 of Monographie BIPM-5 (Bureau International des Poids et Mesures, Pavillon de Breteuil, 92310 Sèvres, France, 2010)

[17] M.-M. Bé, V. Chisté, C. Dulieu, X. Mougeot, V. Chechev, N. Kuzmenko, F. Kondev, A. Luca, M. Galán, A.L. Nichols et al., Table of Radionuclides, Vol. 6 of Monographie BIPM-5 (Bureau International des Poids et Mesures, Pavillon de Breteuil, 92310 Sèvres, France, 2011)

[18] M.-M. Bé, V. Chisté, C. Dulieu, X. Mougeot, V.P. Chechev, F.G. Kondev, A.L. Nichols, X. Huang, B. Wang, Table of Radionuclides, Vol. 7 of Monographie BIPM-5 (Bureau International des Poids et Mesures, Pavillon de Breteuil, 92310 Sèvres, France, 2013)

[19] M.-M. Bé, V. Chisté, C. Dulieu, M.A. Kellett, X. Mougeot, A. Arinc, V.P. Chechev, N.K. Kuzmenko, T. Kibédi, A. Luca et al., Table 
of Radionuclides, Vol. 8 of Monographie BIPM5 (Bureau International des Poids et Mesures, Pavillon de Breteuil, 92310 Sèvres, France, 2016)

[20] T. Kibédi, T.W. Burrows, M.B. Trzhaskovskaya, P.M. Davidson, C.W. Nestor Jr., Nucl. Instrum. Methods Phys. Res. A589, 202 (2008)

[21] X. Mougeot, BetaShape: A new code for improved analytical calculations of beta spectra, in these proceedings (2016)

[22] M.-M. Bé, C. Dulieu, X. Mougeot, M.A. Kellett, Laboratoire National Henri Becquerel - Mini Table of Radionuclides, 2015, ISBN 97-827-598-118-61 (EDP Sciences, 2015)

[23] M.-M. Bé, V.P. Chechev, A. Pearce, Metrologia 52, S66 (2015)

[24] M.-M. Bé, V.P. Chechev, R. Dersch, O.A.M. Helene, R.G. Helmer, M. Herman, S. Hlaváč, A. Marcinkowski, G.L. Molnár, A.L. Nichols et al., Update of X Ray and Gamma Ray Decay Data Standards for Detector Calibration and Other
Applications, IAEA-STI/PUB/1287 (International Atomic Energy Agency, 2007)

[25] R. Capote, A.L. Nichols, F.M. Nortier, B.V. Carlson, A. Hermanne, M. Hussain, A.V. Ignatyuk, M.A. Kellett, T. Kibédi, K. Guinyun et al., IAEA CRP on Nuclear Data for Charged-particle Monitor Reactions and Medical Isotope Production, in these proceedings (2016)

[26] A. Luca, Decay Data Evaluation Project: evaluation of ${ }^{52} \mathrm{Mn}$ and ${ }^{52 m} \mathrm{Mn}$ nuclear decay data, in these proceedings (2016)

[27] V.P. Chechev, Decay Data Evaluation Project (DDEP): updated evaluation of the Ba-140, La-140 and Ce-141 decay characteristics, in these proceedings (2016)

[28] C. Dulieu, M.A. Kellett, X. Mougeot, Decay Data Evaluation Project (DDEP): dissemination and visualisation of reference decay data, in these proceedings (2016)

[29] J.C. Hardy, L.C. Carraz, B. Jonson, P.G. Hansen, Phys. Lett. B 71, 307 (1977) 